\title{
Optimising Pheromone Communication in a UAV Swarm
}

\author{
Daniel H. Stolfi \\ SnT - University of Luxembourg \\ Esch-Sur-Alzette, Luxembourg \\ daniel.stolfi@uni.lu \\ Grégoire Danoy \\ SnT and the FSTM/DCS - University of Luxembourg \\ Esch-Sur-Alzette, Luxembourg \\ gregoire.danoy@uni.lu
}

\author{
Matthias R. Brust \\ SnT - University of Luxembourg \\ Esch-Sur-Alzette, Luxembourg \\ matthias.brust@uni.lu \\ Pascal Bouvry \\ SnT and the FSTM/DCS - University of Luxembourg \\ Esch-Sur-Alzette, Luxembourg \\ pascal.bouvry@uni.lu
}

\begin{abstract}
Communications in multi-robot systems is a key factor, especially when aiming for real-world applications. In this article we address the optimisation of the communications in a swarm of unmanned aerial vehicles for surveillance applications. More precisely a genetic algorithm is introduced to optimize the exchange of pheromone maps used in the CACOC (Chaotic Ant Colony Optimisation for Coverage) mobility model which enhance the vehicles' routes in order to achieve unpredictable trajectories as well as maximise area coverage.
\end{abstract}

\section{CCS CONCEPTS}

-Applied computing $\rightarrow$ Surveillance mechanisms; Aerospace; - Mathematics of computing $\rightarrow$ Combinatorial optimization;

\section{KEYWORDS}

unmanned aerial vehicle, pheromones, evolutionary algorithm, surveillance system, swarm robotics, mobility model

\section{ACM Reference Format:}

Daniel H. Stolfi, Matthias R. Brust, Grégoire Danoy, and Pascal Bouvry. 2021. Optimising Pheromone Communication in a UAV Swarm. In 2021 Genetic and Evolutionary Computation Conference Companion (GECCO '21 Companion), fuly 10-14, 2021, Lille, France. ACM, New York, NY, USA 2 pages. https://doi.org/10.1145/3449726.3459526

\section{INTRODUCTION}

Unmanned Aerial Vehicles (UAVs) initially developed for military applications are nowadays paving their way into multiple civilian domains. Considering surveillance applications, a UAV provides a mobile and controllable bird's-eye view for a fraction of the cost of existing solutions (e.g. helicopters). However, UAVs are typically small to medium size battery-powered devices which therefore feature limited flight time and payload capacity. Using multiple autonomous UAVs simultaneously as a swarm is one promising way of addressing these limitations.

Permission to make digital or hard copies of part or all of this work for personal or classroom use is granted without fee provided that copies are not made or distributed for profit or commercial advantage and that copies bear this notice and the full citation on the first page. Copyrights for third-party components of this work must be honored.

For all other uses, contact the owner/author(s).

GECCO '21 Companion, fuly 10-14, 2021, Lille, France

(C) 2021 Copyright held by the owner/author(s)

ACM ISBN 978-1-4503-8351-6/21/07 . \$15.00

https://doi.org/10.1145/3449726.3459526
In addition, the unpredictability of the vehicles' trajectories in surveillance scenarios is a desired characteristic in order to prevent the use of possible detection strategies. For example, CROMM (Chaotic Rössler Mobility Model) [4], uses chaotic trajectories to avoid route prediction but UAVs tend to visit the same locations frequently. Having this in mind, CACOC (Chaotic Ant Colony Optimisation for Coverage) [4] combines chaos based trajectories with virtual pheromones to spread the swarm throughout the surveillance area. This article proposes to evaluate and optimize the communication range and energy cost within a swarm of UAVs for surveillance missions.

\section{PHEROMONE BASED MOBILITY}

In previous work [5] the optimisation of the CACOC mobility model has been proposed by using three parameters (pheromone amount: $\tau_{a}$, radius: $\tau_{r}$, and scan depth: $\tau_{d}$ ) to adapt it to different scenarios, with the aim of improving area coverage. When using CACOC, UAVs leave virtual pheromones as they move by an environment which is mapped as a lattice of $1 \mathrm{x} 1$ metre cells. The next moving direction is calculated using chaotic dynamics when there are no pheromones in the UAV's neighbourhood, using the first return map from a chaotic attractor [4]. On the other hand, when virtual pheromones are detected, they work as repellers, stochastically modifying the UAV's next moving direction.

Hitherto, $\mathrm{CACOC}$ and its parameterised version $(\mathrm{CACOC}+)$ have been proposed assuming perfect communications [6], where vehicles were always in their respective communication range and no packet was lost. This article proposes to address a more realistic scenario using the ARGoS simulator [3] to take advantage of its communication model between robots.

In CACOC, each vehicle has its own local pheromone map composed by its own pheromone trails and portions of local pheromones maps received from the other vehicles in the simulation scenario (Figure 1). Such local pheromone maps are shared among UAVs via ad hoc communications which are subject to disruptions and interferences. Moreover, larger data packets imply more energy consumption and a higher probability of data loss. This work proposes to optimise CACOC+ parameters where UAVs share different amount of data, and to analyse the impact of partial pheromone maps sharing on the global swarm's performance. Our problem representation is a vector $\vec{x}=\left\{\tau_{a_{1}}, \tau_{r_{1}}, \tau_{d_{1}}, \ldots, \tau_{a_{N}}, \tau_{r_{N}}, \tau_{d_{N}}\right\}$ where $N$ is the number of UAVs in the swarm. It defines the configuration of the surveillance system which comprises the parameters of each UAV in the swarm. 


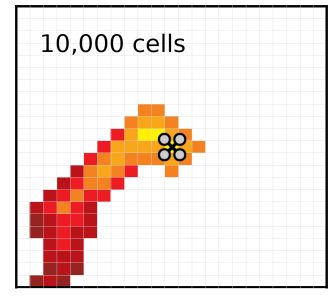

(a) $\mathrm{CACOC}+$

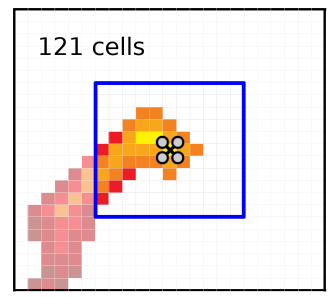

(c) $\mathrm{CACOC}+.05$

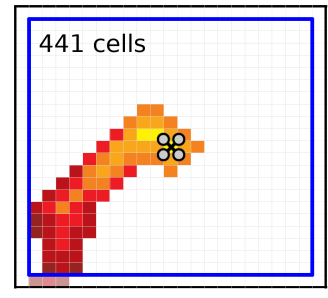

(b) $\mathrm{CACOC}+.10$

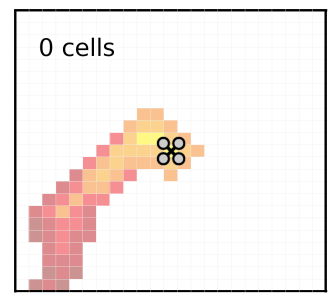

(d) $\mathrm{CACOC}+.00$
Figure 1: Heat map showing the pheromone transmission scenarios in each simulation study.

\section{OPTIMISATION ALGORITHM}

We have designed a Genetic Algorithm (GA) which uses operators for continuous optimisation, in order find the parameterisation of CACOC + which maximizes the area coverage for different data block sizes shared between UAVs. The proposed GA is a generational evolutionary algorithm, whose operators are: binary tournament [2], uniform crossover which works with UAV configuration blocks, a mutation operator based on [1] and adapted to our problem characteristics, and as replacement operator, the worst individual of the population is replaced by the best one in the working population to avoid stagnation and preserve diversity [1].

Each individual representing a vehicles' configuration is evaluated through an ARGoS simulation. Each simulation scenario is mapped as a lattice of $100 \times 100$ cells for evaluation purpose, and it is assumed that a UAV explores an area of $3 \times 3$ cells at each simulation tick. Consequently, the fitness value of a given configuration is calculated as $F(\vec{x})=\frac{\text { \# of explored cells }}{\text { \# of cells in the scenario }}$. The objective is to maximise exploration, thus the higher the value of $F(\vec{x})$, the better.

\section{EXPERIMENTAL RESULTS}

The optimisation of three simulation studies has been first conducted using CACOC+ as mobility model, by performing 30 independent runs of the proposed GA. Each simulation study consists in four simulation scenarios where swarms of two, four and six UAVs are analysed. In CACOC+ the entire pheromone map is shared between UAVs in communication range (10 metres). In CACOC +.10 a square of $21 \mathrm{x} 21$ cells is shared, in CACOC +.05 a square of $11 \mathrm{x} 11$ cells is shared, and in CACOC +.00 no pheromone map is shared between UAVs (Figure 1).

In a second stage, the $\mathrm{CACOC}+$ area coverage performance was compared to CACOC and CROMM. Table 1 shows the area coverage values achieved by those mobility models where it can be seen that the UAVs using CACOC+ cover more area than CACOC. When the
UAVs controlled by CACOC were subject to the same communication restrictions as $\mathrm{CACOC}+$, their coverage values showed bigger variability, being notably affected by the amount of pheromones shared between them, especially when there are more pheromones in the simulation scenario (more UAVs). The lowest CROMM coverage values confirm the need of virtual pheromones as a complement of chaotic mobility to improve the area coverage of the system.

Table 1: Area coverage achieved in each simulation scenario.

\begin{tabular}{|c|c|c|c|}
\hline Simulation Study & $2 \mathrm{UAV}$ & $4 \mathrm{UAV}$ & $6 \mathrm{UAV}$ \\
\hline CROMM & $13.3 \%$ & $43.5 \%$ & $52.2 \%$ \\
\hline$\overline{\mathrm{C}} \mathrm{A} \overline{\mathrm{CO}} \overline{\mathrm{C}}$ & $\overline{4} 6 . \overline{5 \%}$ & $\overline{71} . \overline{0} \%$ & $8 \overline{5} .8 \overline{\%}$ \\
\hline CACOC.10 & $46.5 \%$ & $68.7 \%$ & $85.8 \%$ \\
\hline CACOC.05 & $46.5 \%$ & $71.6 \%$ & $84.2 \%$ \\
\hline CACOC.00 & $38.8 \%$ & $70.2 \%$ & $80.0 \%$ \\
\hline$\overline{\mathrm{C}} \overline{\mathrm{AC}} \overline{\mathrm{O}} \overline{\mathrm{C}}+$ & $\overline{5} 3 . \overline{8} \%$ & $\overline{78} . \overline{0} \%$ & $-8 \overline{9} .5 \overline{\%}$ \\
\hline $\mathrm{CACOC}+.10$ & $53.5 \%$ & $78.8 \%$ & $89.3 \%$ \\
\hline $\mathrm{CACOC}+.05$ & $53.5 \%$ & $78.2 \%$ & $89.3 \%$ \\
\hline $\mathrm{CACOC}+.00$ & $53.9 \%$ & $78.6 \%$ & $89.6 \%$ \\
\hline
\end{tabular}

\section{CONCLUSIONS AND FUTURE WORK}

This article introduced the optimisation of the CACOC+ mobility model, initially proposed to improve area coverage, with the aim of obtaining good performance values when pheromone data is shared between UAVs using realistic communications. The simulator ARGoS was used in our experimentation to implement the mobility and data transmission models. Experimental results demonstrate that $\mathrm{CACOC}+$ improves $\mathrm{CACOC}$ and $\mathrm{CROMM}$ also under these new conditions and that the versatility of the UAVs parameterisation has compensated the reduced knowledge of the pheromone neighbourhood of each UAV.

\section{ACKNOWLEDGMENTS}

This work relates to Department of Navy award N62909-18-1-2176 issued by the Office of Naval Research. The United States Government has a royaltyfree license throughout the world in all copyrightable material contained herein. This work is partially funded by the joint research programme UL/SnT-ILNAS on Digital Trust for Smart-ICT.

\section{REFERENCES}

[1] R. Chelouah and P. Siarry. 2000. Continuous genetic algorithm designed for the global optimization of multimodal functions. Fournal of Heuristics 6, 2 (2000), 191-213. https://doi.org/10.1023/A:1009626110229

[2] David E Goldberg. 1989. Genetic Algorithms in Search, Optimization and Machine Learning (1st ed.). Addison-Wesley Longman Publ Co., Inc., Boston, MA, USA.

[3] Carlo Pinciroli, Vito Trianni, Rehan O'Grady, Giovanni Pini, Arne Brutschy, Manuele Brambilla, Nithin Mathews, Eliseo Ferrante, Gianni Di Caro, Frederick Ducatelle, Mauro Birattari, Luca Maria Gambardella, and Marco Dorigo. 2012. ARGoS: a modular, parallel, multi-engine simulator for multi-robot systems. Swarm Intelligence 6, 4 (dec 2012), 271-295. https://doi.org/10.1007/s11721-012-0072-5

[4] Martin Rosalie, Grégoire Danoy, Serge Chaumette, and Pascal Bouvry. 2018. Chaosenhanced mobility models for multilevel swarms of UAVs. Swarm and Evol. Comput. 41, November 2017 (2018), 36-48. https://doi.org/10.1016/j.swevo.2018.01.002

[5] Daniel H. Stolfi, Matthias R. Brust, Gregoire Danoy, and Pascal Bouvry. 2020. A Cooperative Coevolutionary Approach to Maximise Surveillance Coverage of UAV Swarms. In 2020 IEEE 17th Annual Consumer Communications \& Networking Conference (CCNC). IEEE, 1-6. https://doi.org/10.1109/ccnc46108.2020.9045643

[6] Daniel H. Stolfi, Matthias R. Brust, Gregoire Danoy, and Pascal Bouvry. 2020. Competitive Evolution of a UAV Swarm for Improving Intruder Detection Rates. In 2020 IEEE International Parallel and Distributed Processing Symposium Workshops (IPDPSW). IEEE, 528-535. https://doi.org/10.1109/IPDPSW50202.2020.00094 